\title{
Systematic review and meta-analysis of the effects of the perioperative enhanced recovery after surgery concept on the surgical treatment of acute appendicitis in children
}

\author{
Anping Zhang ${ }^{1 \#}$, Hao Lu ${ }^{2 \#}$, Fangfang Chen ${ }^{1}$, You $\mathrm{Wu}^{3}$, Liqiong Luo ${ }^{2}$, Siyi Sun ${ }^{1}$ \\ ${ }^{1}$ Anesthesia Surgery Department, Sichuan Provincial Maternity and Child Health Care Hospital, Chengdu, China; ${ }^{2}$ Children's Heart Center, Sichuan \\ Provincial Maternity and Child Health Care Hospital, Chengdu, China; ${ }^{3}$ Pediatric Surgery Department, Sichuan Provincial Maternity and Child \\ Health Care Hospital, Chengdu, China \\ Contributions: (I) Conception and design: A Zhang, H Lu, S Sun; (II) Administrative support: F Chen; (III) Provision of study materials or patients: A \\ Zhang, F Chen, Y Wu, L Luo, S Sun; (IV) Collection and assembly of data: All authors; (V) Data analysis and interpretation: A Zhang, H Lu, Y Wu, \\ L Luo; (VI) Manuscript writing: All authors; (VII) Final approval of manuscript: All authors. \\ \#These authors contributed equally to this work. \\ Correspondence to: Siyi Sun. Anesthesia Surgery Department, Sichuan Provincial Maternity and Child Health Care Hospital, No. 290, Shayan West \\ Second Street, Wuhou District, Chengdu, China. Email: 18908098262@126.com.
}

Background: Enhanced recovery after surgery (ERAS), as a new concept in surgery, has dramatically changed the mode of perioperative treatment for children with acute appendicitis.

Methods: The retrieval strategy developed by the Cochrane Collaboration was conducted using the CNKI database, Wanfang Medical Network, PubMed, EBSCO, Medline, and Cochrane database by combining subject headings and free words. A review of the randomized controlled trials on the use of the ERAS concept in the perioperative treatment of acute appendicitis in children was conducted between the establishment of the database and May 15, 2021. Keywords included enhanced recovery after surgery, fast track surgery, ERAS, FTS, child, infant, and appendicitis. The quality of the literature was evaluated according to the RevMan 5.3 software provided by the Cochrane Collaboration.

Results: Five randomized controlled trials on ERAS in children with acute appendicitis were finally included. The heterogeneity of postoperative stay time was tested in 4 studies using continuous variables, with Chi-squared test $\left(\mathrm{Chi}^{2}\right)=221.52$, degree of freedom $(\mathrm{df})=3, \mathrm{I}^{2}=99 \%>50 \%$. An overall analysis using a random effects model showed that the ERAS group was significantly different compared to the control group $[\mathrm{Z}=5.26$; mean difference $(\mathrm{MD})=-1.65 ; 95 \% \mathrm{CI}:-2.27$ to $-1.03 ; \mathrm{P}<0.00001]$. The heterogeneity of the readmission rate was tested in 5 studies using dichotomous variables, with $\mathrm{Chi}^{2}=5.11, \mathrm{df}=3, \mathrm{I}^{2}=41 \%<50 \%$, $\mathrm{P}=0.91$. Overall analysis using a fixed effects model showed no statistically significant difference between the ERAS group and the control group $[\mathrm{Z}=0.80$; odds ratio $(\mathrm{OR})=1.16$; $95 \% \mathrm{CI}$ : 0.81 to 1.66 ; $\mathrm{P}=0.42]$. The heterogeneity of the recurrence rate was tested in 4 studies using dichotomous variables, with $\mathrm{Chi}^{2}=3.73$, df $=3, \mathrm{I}^{2}=20 \%<50 \%, \mathrm{P}=0.29$. Overall analysis using a fixed effects model showed no statistically significant difference between the ERAS group and the control group $(\mathrm{Z}=1.14$; OR $=0.76$; $95 \% \mathrm{CI}$ : 0.47 to 1.22 ; $\mathrm{P}=0.26)$.

Discussion: The results of the meta-analysis confirmed that perioperative application of the ERAS concept in children with acute appendicitis can promote the rehabilitation of children, reduce the postoperative stay time, and reduce the readmission rate and reoperation rate.

Keywords: Enhanced recovery after surgery (ERAS); acute appendicitis in children; perioperative period; treatment; meta-analysis

Submitted Sep 09, 2021. Accepted for publication Nov 03, 2021.

doi: $10.21037 / \mathrm{tp}-21-457$

View this article at: https://dx.doi.org/10.21037/tp-21-457 


\section{Introduction}

Acute appendicitis in children is one of the most common acute abdominal diseases. Its clinical signs and symptoms include periumbilical pain, metastatic right lower abdominal pain, rebound pain, vomiting, diarrhea, fever, and elevated blood counts (1). The younger the child, the more atypical the symptoms, the more difficult the diagnosis, and the higher the perforation rate $(2,3)$. The characteristics of the disease include its occult and rapid onset, rapid progression, and diagnostic difficulty. If treatment and diagnosis are delayed, there can be serious consequences. The first diagnosis is usually in the department of pediatrics. Due to the imperfect development of the omentum in infants, diffuse peritonitis can easily occur when the appendix has inflammation and perforation (4). Compared with adult acute appendicitis, which typically presents with metastatic right lower abdominal pain, infants are specific and difficult to diagnose. Wilmore et al. [2001] (5) confirmed that the incidence of perforation was $>65 \%$ if children failed to visit the doctor within 48 hours, which has a significant impact on children's physical and mental health and quality of life. Early diagnosis and treatment are key to improving the prognosis of infants with acute appendicitis (6).

The enhanced recovery after surgery (ERAS) concept is a new surgical theory proposed by surgeons Patkova et al. (7). By taking a series of medically recognized measures during the perioperative period, patients' traumatic stress in terms of physical and psychological aspects can be reduced. It is a clinical practice to reduce the incidence of postoperative complications, shorten the recovery time, reduce the number of days in hospital, and reduce the cost of hospitalization. It mainly includes fast-track anesthesia, minimally invasive techniques, and reasonable analgesia, so that patients can recover quickly. The clinical effect of surgical treatment of acute appendicitis is very significant. However, in the treatment process, the intensive nursing care of patients during perioperative period is often neglected, which leads to the increase of complications, seriously affects the prognosis of patients and enhances the pain of patients. With the continuous progress of medical technology, more and more attention has been paid to nursing intervention in clinic. Nursing intervention before, during and after operation for perioperative patients with acute appendicitis can significantly reduce the incidence of complications and improve nursing satisfaction. Compared with the traditional method, getting out of bed early can maintain the muscle function after operation. Early postoperative oral nutrition intake can reduce the damage of postoperative pulmonary function, restore gastrointestinal peristalsis as soon as possible, reduce activity and enhance cardiovascular function. As a new surgical concept, accelerated surgery has greatly changed the perioperative treatment of many surgical operations $(8,9)$. However, accelerated rehabilitation surgery has been gradually applied by surgeons in clinical practice, and its safety and effectiveness in treating acute appendicitis in children have yet to be confirmed.

This meta-analysis aimed to explore the safety and efficacy of accelerated rehabilitation surgery in the perioperative treatment of acute appendicitis in children.

We present the following article in accordance with the PRISMA reporting checklist (available at https://dx.doi. org/10.21037/tp-21-457).

\section{Methods}

\section{Literature search}

The retrieval strategy developed by the Cochrane Collaboration was conducted using the CNKI database, Wanfang Medical Network, PubMed, EBSCO, Medline, and Cochrane database by combining subject headings and free words. Randomized controlled trials on the application of the accelerated rehabilitation surgical concept in the perioperative treatment of acute appendicitis in children published between database establishment and May 15, 2021 were retrieved. The keywords in English were: enhanced recovery after surgery, fast track surgery, ERAS, FTS, child, infant, and appendicitis. The Chinese search keywords included: rapid recovery, accelerated recovery, children, infantile, infant, and appendicitis. The quality of the literature was evaluated according to the RevMan 5.3 software provided by the Cochrane Collaboration.

The above search terms were freely combined, and the references that could be included were obtained after multiple searches. Then, a search engine was used to trace each document, and finally the latest research progress was obtained by contacting the relevant experts and researchers of the published literature.

\section{Literature inclusion and exclusion criteria}

The included literature met the following criteria: (I) domestic and foreign published randomized controlled trials on the clinical effect of the perioperative accelerated 
rehabilitation surgery concept in the treatment of acute appendicitis in children (3-18 years old) versus traditional treatment; (II) the subjects were children with clinically diagnosed acute appendicitis; (III) pathological control analysis, with the index comparison reliable at the $95 \%$ confidence interval (CI); (IV) the research hypothesis and research methods of each study were similar, and there was a clear publication period.

Articles characterized by any of the following were excluded: (I) age ranged from 3 to 18 years; (II) children with appendicitis perforation, gangrene, and abscess; (III) non-Chinese and English documents; (IV) repeated published studies, case reports, lectures, and reviews; (V) after contacting the original author of the literature, complete data could not be obtained.

\section{Outcome indicators}

The outcome indexes were postoperative stay time, complications, incision infection rate, postoperative residual abdominal abscess rate, complication rate, readmission rate and reoperation rate.

\section{Data extraction}

Two evaluators used a unified Microsoft Excel spreadsheet (Microsoft, the United States) to independently conduct literature screening and data extraction, which was then cross-checked. If any disagreement was encountered, it would be resolved through discussion. The main extracted data included: (I) the basic information of the study, such as the title of the study, the name of the first author, the publication year, the journal of publication; (II) the basic characteristics of the study subjects, such as sex, age, number of cases, etc.; (III) specific operation of intervention measures and follow-up time; (IV) key factors of bias risk; (V) the outcome indicators and outcome data were extracted, including postoperative stay time, complications, incision infection rate, postoperative residual abdominal abscess rate, complication rate, readmission rate and reoperation rate.

\section{Bias risk assessment}

The risk of bias in the included randomized controlled trials was assessed by two researchers at the same time, and the results were determined by discussion if the two disagreed. In this study, the Cochrane manual was used as the tool for "risk assessment of bias" in the randomized controlled trials. The evaluation criteria included case selection, trials to be evaluated, gold standard, pathological procedures, and pathological progress. "High risk bias", "low risk bias", and "unclear" were judged for the above five aspects.

\section{Quality assessment}

In this study, the GRADE standard of the Cochrane Collaboration was used for quality classification. Literature with a score of 2 or below was considered to be of low quality, that is, high-risk bias. A score of 3-5 was considered medium quality. A score of 6-9 was considered high quality.

\section{Sensitivity analysis}

The results were compared by the random effects model and fixed effects model, and the reliability of the combined results was analyzed according to the consistency degree of the results. A funnel plot was used to determine whether publication bias existed.

\section{Statistical analysis}

Stata SE12.0 software was used for statistical analysis. The odds ratio (OR) was used as the dichotomous variable, and the mean difference (MD) was used as the continuous variable. The risk of bias of the included studies was assessed using the bias risk assessment graph of RevMan 5.3 software. The data were sorted, screened, and input into RevMan 5.3 software. Results and charts were obtained after analysis. All effects were expressed by the $95 \%$ CI. When $\mathrm{P}>0.01$ and $\mathrm{I}^{2}<50 \%$, the fixed effects model was used for meta-analysis. When $\mathrm{P}<0.01$ and $\mathrm{I}^{2}>50 \%$, the random effects model was used for meta-analysis.

\section{Results}

\section{Search results and basic information of the literature}

304 documents were retrieved from the database, 132 documents were retrieved from the register, 87 documents published twice were eliminated, 52 documents were eliminated from reading topics and abstracts, and 28 documents were eliminated for other reasons, leaving 269 documents. After eliminating 147 papers and 71 papers, there are 51 papers left. Because of the review, incomplete outcome index, and research objects, 46 papers were 


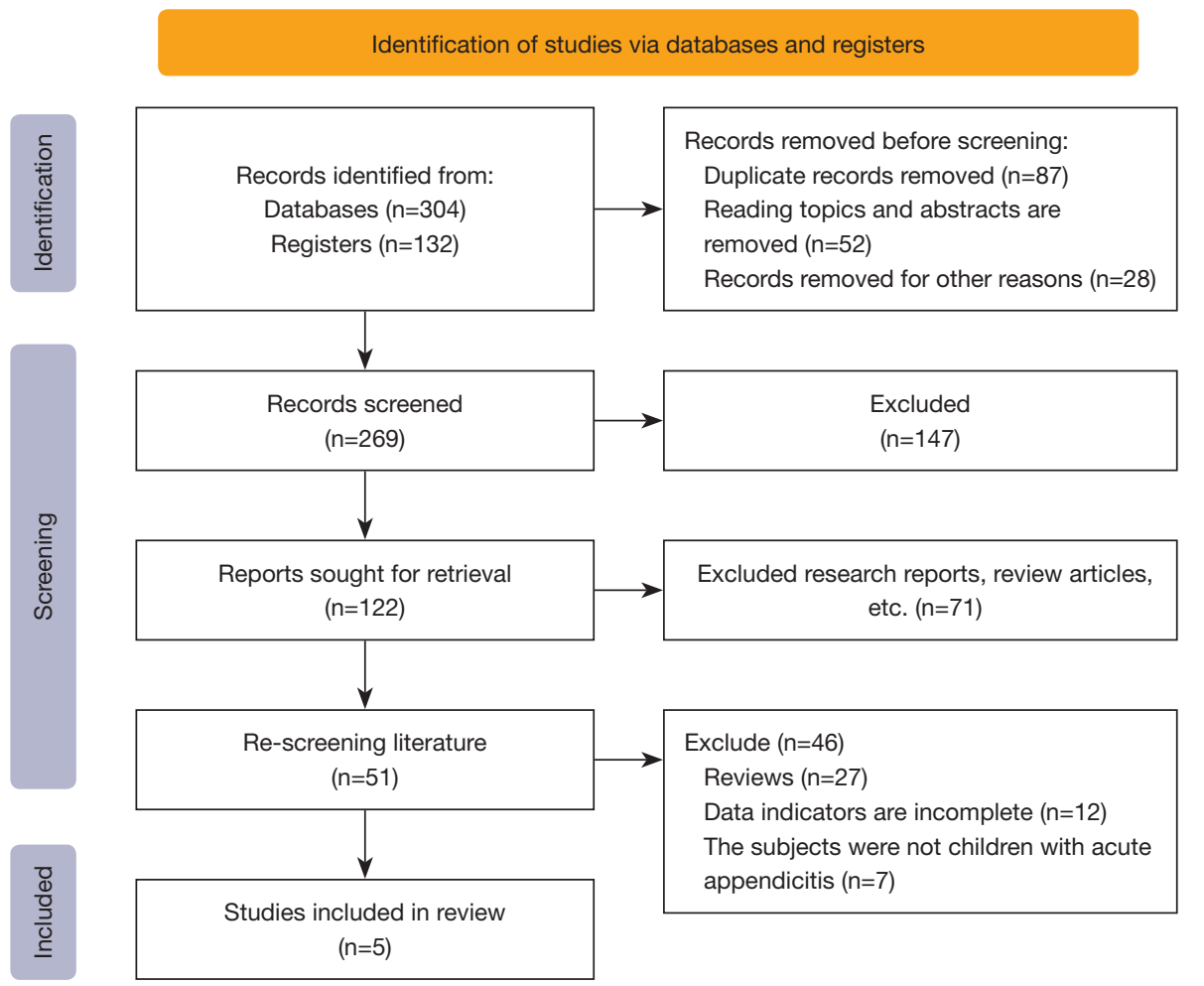

Figure 1 Literature screening process.

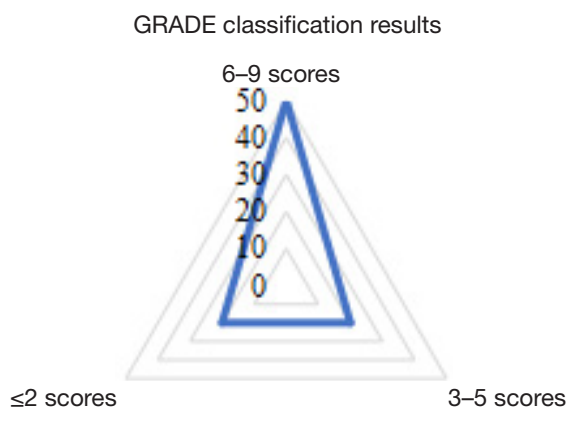

Figure 2 GRADE classification results.

excluded, and finally 5 papers were included for metaanalysis. Figure 1 is a flowchart depicting the literature retrieval and screening process.

Figure 2 shows the results of GRADE classification. It can be seen that there were 3 references with scores of 6-9 (60\%), 1 reference with scores of 3-5 (20\%), and 1 reference with a score below $1(20 \%)$.

There were 5 studies meeting the inclusion criteria, and 2,402 patients were counted. The 5 studies were all small sample studies, in which the sample size ranged from 70 to
1,745 cases, and the age of the study subjects ranged from 3 to 18 years old. The postoperative stay time, complications, incision infection rate, postoperative residual abdominal abscess rate, complication rate, readmission rate and reoperation rate of patients in the experimental group and control group were described in detail. Table 1 shows the basic characteristics of the included literature.

\section{Results of the risk of bias evaluation of the included literature}

Figure 3 and Figure 4 show the multiple risk of bias evaluation results of the studies plotted by RevMan 5.3 software. In this study, among the 5 randomized controlled trials, 2 randomized controlled trials described the correct random allocation method, accounting for $40 \%$, and only 1 randomized controlled trial described the allocation concealment scheme in detail, accounting for $20 \%$. Blinding was not used in the rest of the papers, but the measurement indexes in the studies were laboratory indexes determined by computer, so it can be considered that blinding was correctly used in all the papers. 


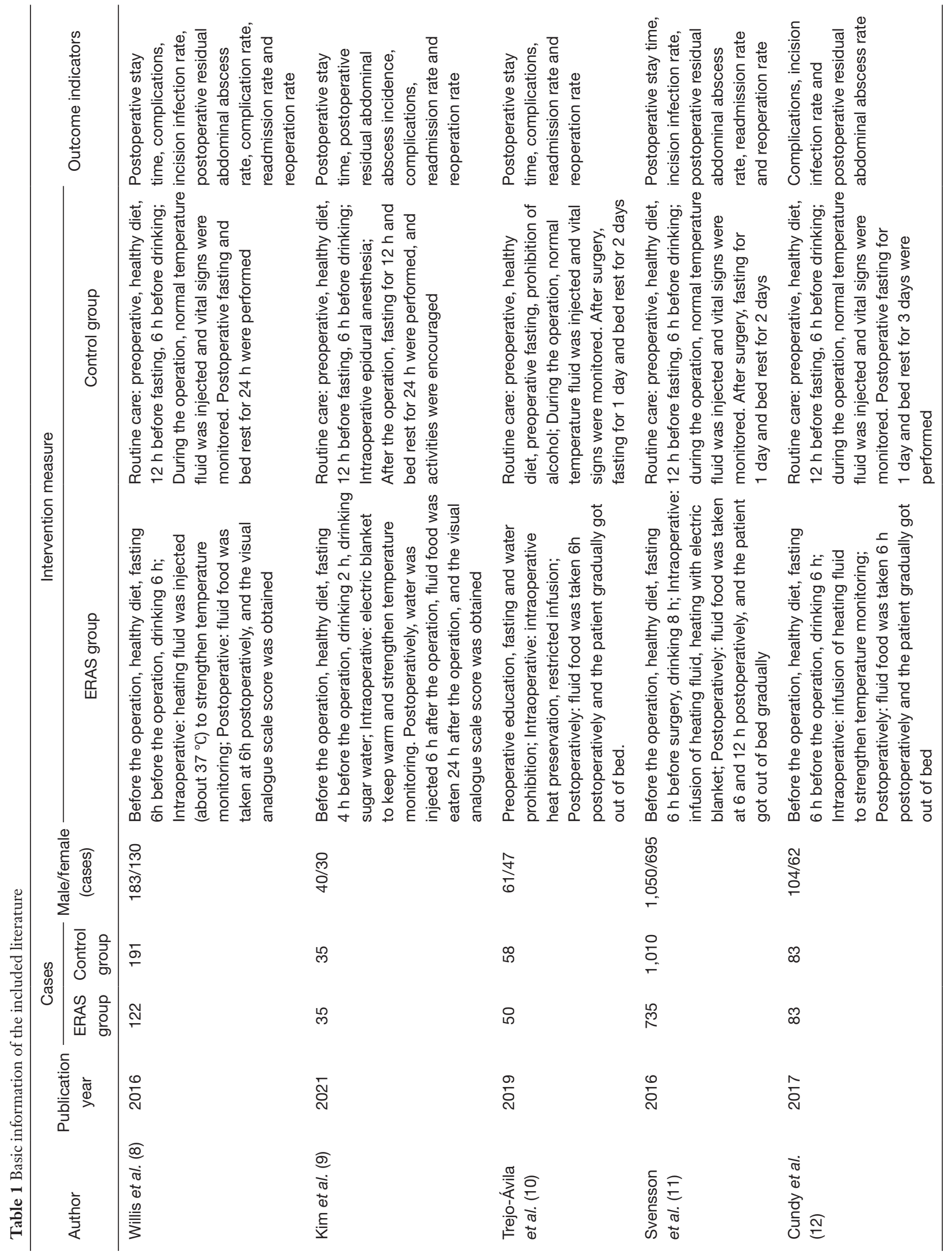




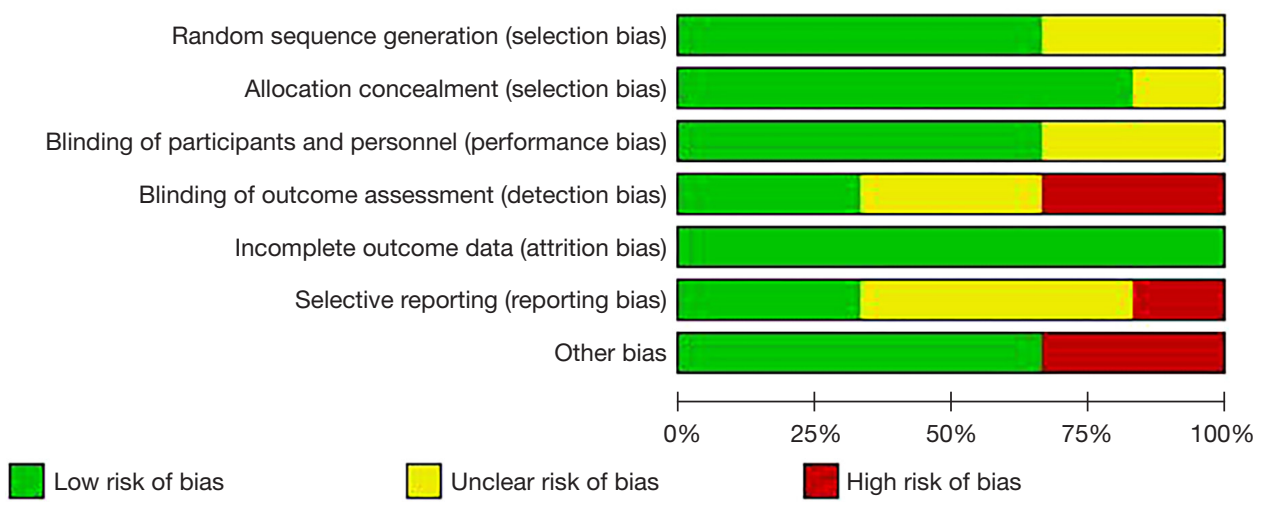

Figure 3 Literature risk of bias evaluation results.

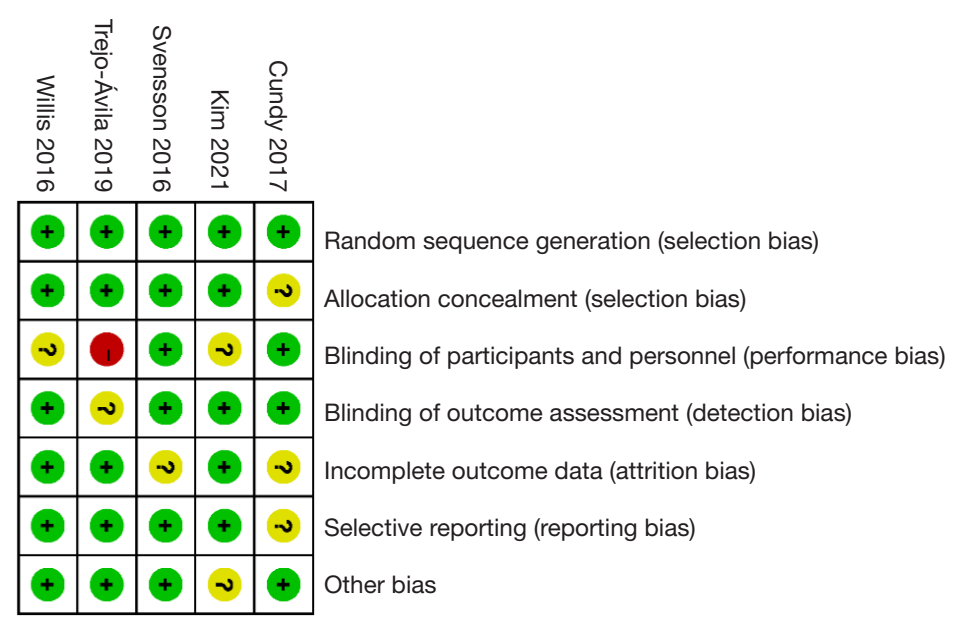

Figure 4 The bias-risk assessment diagram of the included articles.

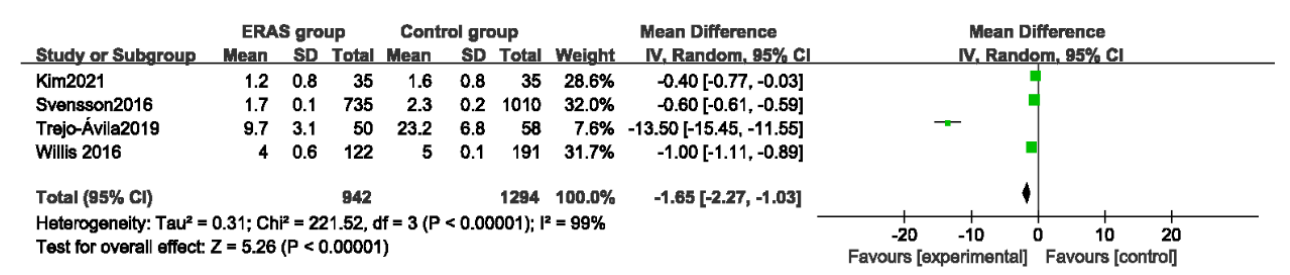

Figure 5 Forest plot of the fixed effects model for postoperative stay time.

\section{Meta-analysis of postoperative stay time}

A total of 4 randomized controlled trials analyzed the length of postoperative stay time. Figure 5 shows the forest plot of the random effects model for postoperative stay time. In the 4 studies, continuous variables were used to describe postoperative stay time, with a total of 2,236 cases, including 942 cases in the ERAS group and 1,294 cases in the control group. Overall heterogeneity was tested, with $\mathrm{Chi}^{2}$ (Chi-squared test $)=221.52, \mathrm{df}($ degrees of freedom $)=3$, $\mathrm{I}^{2}=99 \%>50 \%$. In addition, the horizontal lines of the $95 \%$ CIs in all studies were to the left of the invalid vertical line. An overall analysis using a random effects model showed that the ERAS group was significantly different compared to the control group $(\mathrm{Z}=5.26 ; \mathrm{MD}=-1.65 ; 95 \% \mathrm{CI}:-2.27$ to $-1.03 ; \mathrm{P}<0.00001)$. 


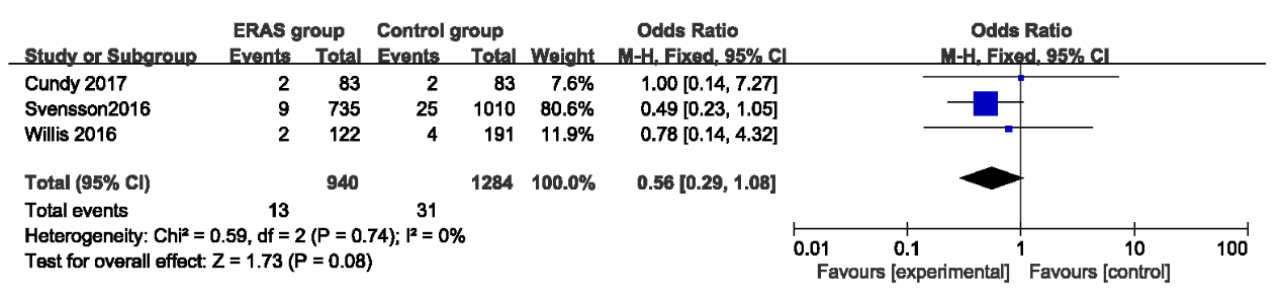

Figure 6 Forest plot of the fixed effects model for postoperative incision infection rate.

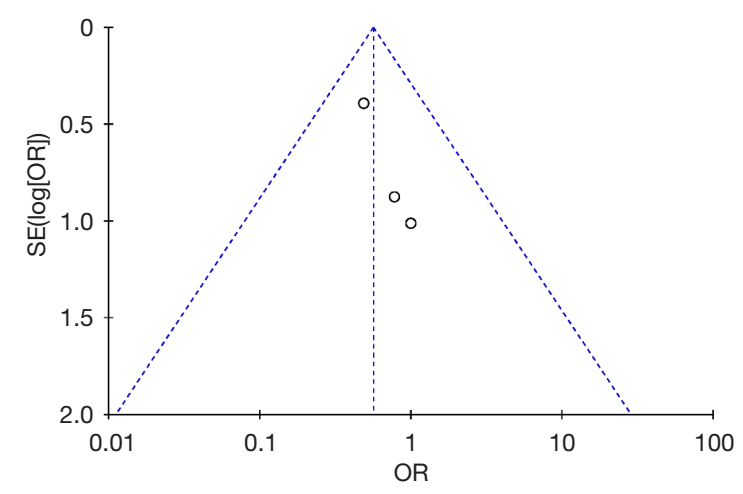

Figure 7 Funnel plot of postoperative incision infection rate.

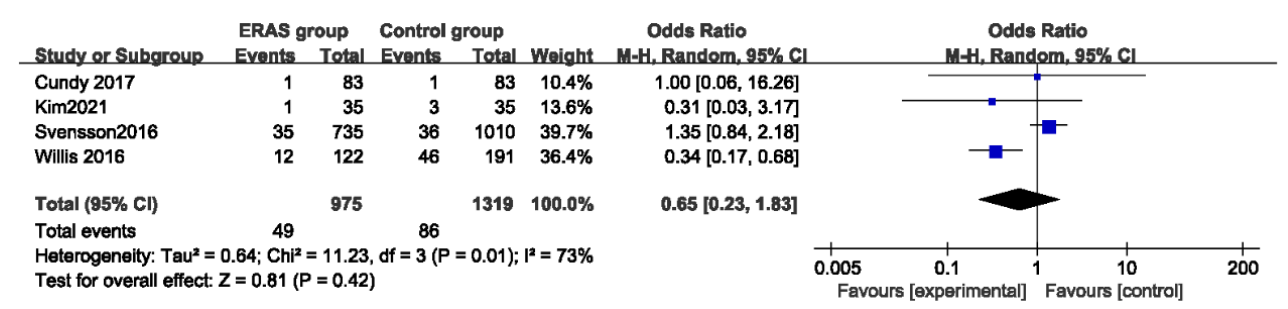

Figure 8 Forest plot of the fixed effects model for incidence of postoperative residual abdominal abscess.

\section{Meta-analysis of the postoperative incision infection rate}

A total of 3 randomized controlled trials analyzed postoperative incision infection rates. Figure 6 shows the forest plot of the fixed effects model for postoperative incision infection rate. In the 3 studies, the postoperative incision infection rate was described using dichotomous variables. There were a total of 2,224 cases, including 940 cases in the ERAS group and 1,284 cases in the control group. The overall heterogeneity was tested, with $\mathrm{Chi}^{2}=0.59, \mathrm{df}=2, \mathrm{I}^{2}=0 \%<50 \%, \mathrm{P}=0.74>0.01$. Overall analysis using a fixed effects model showed that the ERAS group was not significantly different compared to the control group $(\mathrm{Z}=1.73 ; \mathrm{OR}=0.56 ; 95 \% \mathrm{CI}$ : 0.29 to $1.08 ; \mathrm{P}=0.08)$.

Figure 7 is a funnel chart of incision infection rate. It can be seen that the included literatures are concentrated near the midline and are basically symmetrical, indicating that there is no publication bias in the included literatures.

\section{Meta-analysis of the incidence of postoperative residual abdominal abscess}

A total of 4 randomized controlled trials analyzed the incidence of postoperative residual abdominal abscess, as shown in Figure 8 . In the 4 studies, the the incidence of postoperative residual abdominal abscess was described using binary variables. The total number of cases was 2,294, including 975 in the ERAS group and 1,319 in the control group. The overall heterogeneity was tested, with $\mathrm{Chi}^{2}=11.23, \mathrm{df}=3, \mathrm{I}^{2}=73 \%>50 \%, \mathrm{P}=0.01$. The overall 
analysis using the random effects model showed no statistically significant difference between the ERAS group and the control group ( $\mathrm{Z}=0.81$; $\mathrm{OR}=0.65 ; 95 \% \mathrm{CI}: 0.23$ to $1.83 ; \mathrm{P}=0.42$ ).

Figure 9 is a funnel chart of the incidence of postoperative residual abdominal abscess. It can be seen that the included literatures are concentrated near the midline, which indicates that there is no publication bias in the included literatures.

\section{Meta-analysis of the complication rate}

A total of 4 randomized controlled trials analyzed the complication rate, as shown in Figure 10. In the 4 studies, the complication rate was described using binary variables. The total number of cases was 657, including 290 in the ERAS group and 367 in the control group. The overall heterogeneity was tested, with $\mathrm{Chi}^{2}=0.20 \mathrm{df}=2$, $\mathrm{I}^{2}=0 \%<50 \%, \mathrm{P}=0.91$. The overall analysis using the fixed effects model showed no statistically significant difference between the ERAS group and the control group $(\mathrm{Z}=1.80$, $\mathrm{OR}=0.63 ; 95 \%$ CI: 0.38 to $1.04 ; \mathrm{P}=0.07$ ).

Figure 11 is a funnel chart of the incidence of complications. It can be seen that the included literatures

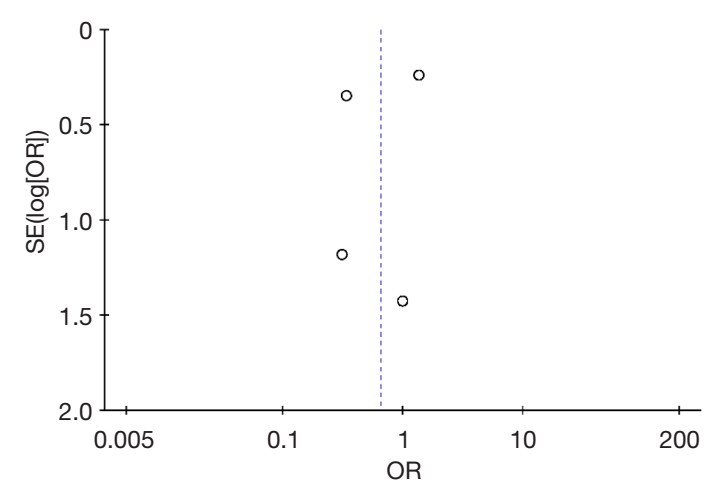

Figure 9 Funnel plot of incidence of postoperative residual abdominal abscess. are concentrated near the midline, which indicates that there is no publication bias in the included literatures.

\section{Meta-analysis of the readmission rate}

A total of 5 randomized controlled trials analyzed the readmission rate (Figure 12). The 5 studies used dichotomous variables to describe the readmission rate. There were 2,402 cases in total, with 1,025 cases in the ERAS group and 1,377 cases in the control group. The overall heterogeneity was tested, with $\mathrm{Chi}^{2}=5.11, \mathrm{df}=3$, $\mathrm{I}^{2}=41 \%<50 \%, \mathrm{P}=0.16$. The overall analysis using a fixed effects model showed no statistically significant difference between the ERAS group and the control group $(\mathrm{Z}=0.80$; $\mathrm{OR}=1.16 ; 95 \%$ CI: 0.81 to $1.66 ; \mathrm{P}=0.42$ ).

Figure 13 is a funnel chart of readmission rate. It can be seen that the included literatures are concentrated near the midline, which indicates that there is no publication bias in the included literatures.

\section{Meta-analysis of the recurrence rate}

A total of 4 randomized controlled trials analyzed the

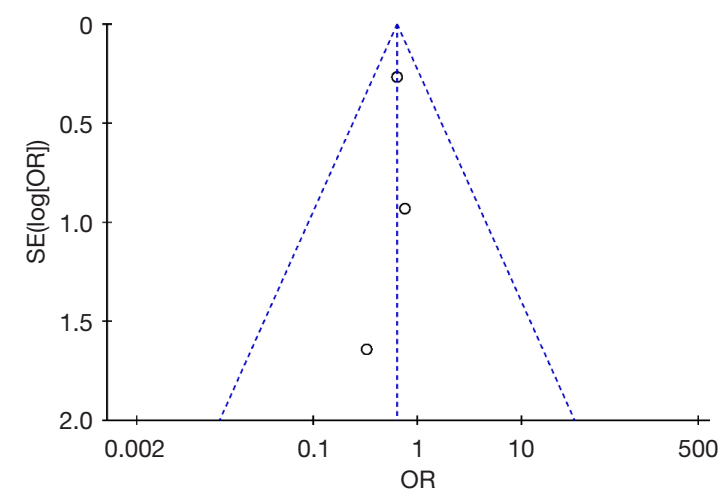

Figure 11 Funnel plot of complication rate.

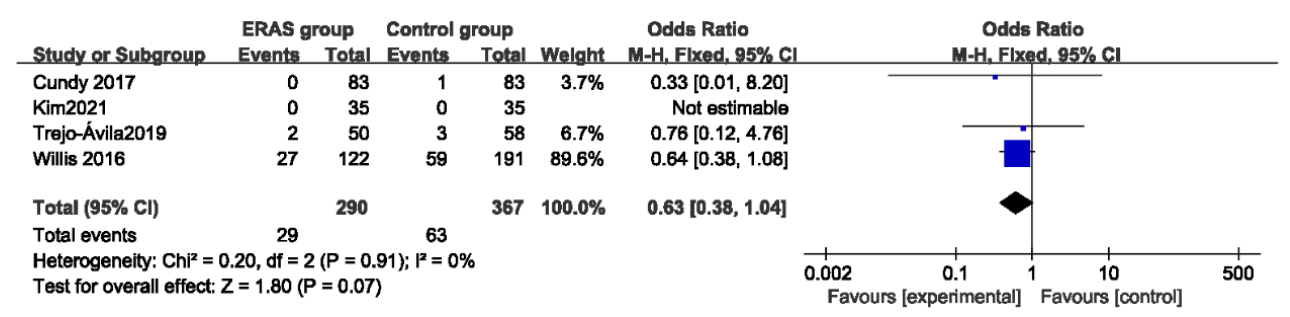

Figure 10 Forest plot of the fixed effects model for complication rate. 


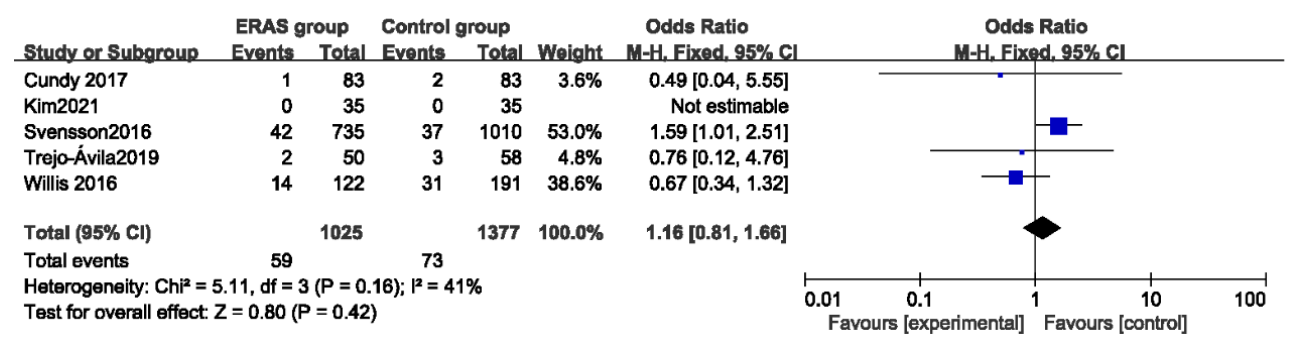

Figure 12 Forest plot of the fixed effects model for readmission rate.

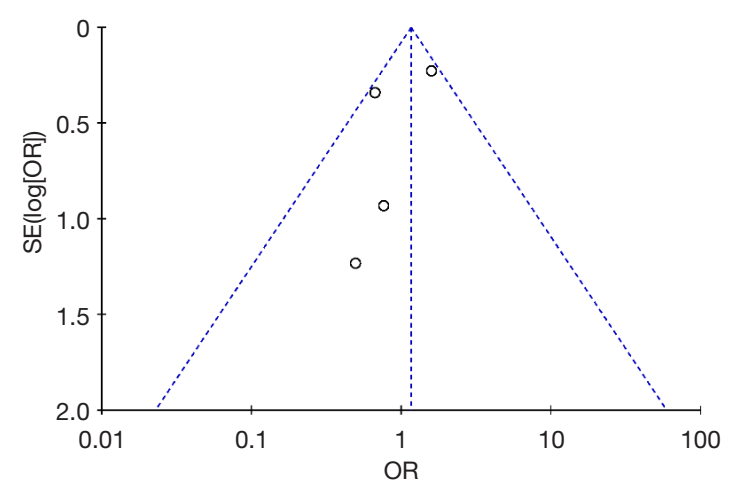

Figure 13 Funnel plot of readmission rate.

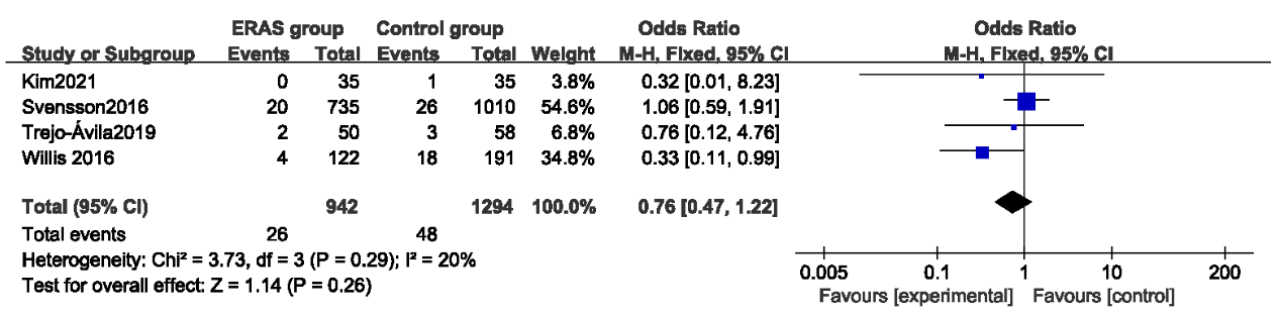

Figure 14 Forest plot of the fixed effects model for recurrence rate.

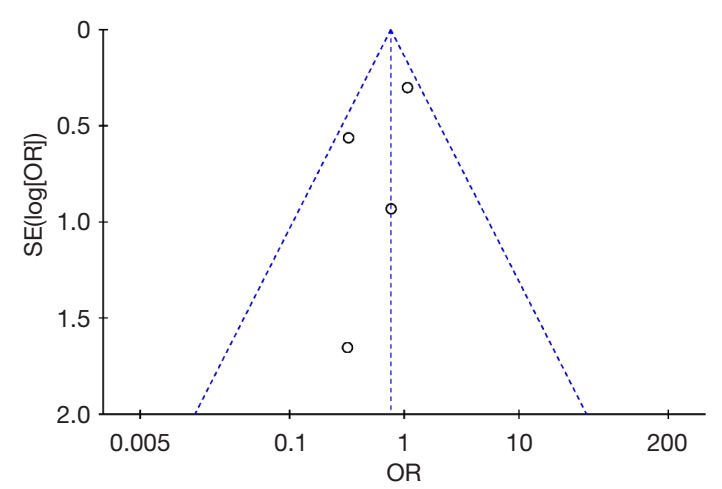

Figure 15 Funnel plot of recurrence rate. recurrence rate (Figure 14). The 4 studies used dichotomous variables to describe the recurrence rate. There were 2,236 cases in total, with 942 cases in the ERAS group and 1,294 cases in the control group. The overall heterogeneity was tested, with $\mathrm{Chi}^{2}=3.73$, df $=3, \mathrm{I}^{2}=20 \%<50 \%, \mathrm{P}=0.29$. The overall analysis using a fixed effects model showed no statistically significant difference between the ERAS group and the control group $(\mathrm{Z}=1.14$; $\mathrm{OR}=0.76,95 \% \mathrm{CI}: 0.47$ to 1.22; $\mathrm{P}=0.26)$.

Figure 15 is a funnel chart of reoperation rate. It can be seen that the included literatures are basically symmetrical around the midline, which indicates that there is no publication bias in the included literatures. 


\section{Sensitivity and publication bias analysis}

The random effect model is used to calculate the postoperative stay time, and the comprehensive results are highly reliable. The fixed effect model was also used to calculate the postoperative incision infection rate, postoperative abdominal residual abscess rate, complication rate, readmission rate and reoperation rate. The results show that the reliability of each outcome index is high. It can be seen from the funnel diagram that the circles and midlines included in the study are basically symmetrical, which indicates that the study is highly accurate, the publication is unbiased, and the final conclusion is relatively credible.

\section{Discussion}

Since the beginning of the 21st century, the medical model has been gradually transformed from the pure biological model to the physio-psycho-medical model, and the requirements of rapid recovery for surgical patients have gradually increased. The ERAS concept has emerged on this basis (13-15). ERAS takes the patient as the center, adopts the optimized perioperative treatment, alleviates the stress response caused by surgery, reduces the postoperative complication rate, and reduces the length of hospital stay, thus achieving the aim of rapid recovery (16-18). ERAS involves a combination of rehabilitation, surgery, anesthesia, nursing, and other disciplines to optimize routine perioperative treatment (19). Using the concept of rapid rehabilitation surgery to guide patients undergoing gynecological laparoscopic surgery, early drinking, early eating and early voluntary activities can promote the recovery of gastrointestinal function and shorten the first exhaust time after surgery. It is of high clinical value to implement the concept of rapid rehabilitation surgery to guide nursing care for patients undergoing cardiac surgery, which can obviously promote the prognosis of patients. The concept of rapid rehabilitation surgery for patients undergoing gastrointestinal surgery during hospitalization can significantly reduce the trauma and stress of patients during perioperative period, and accelerate the recovery of patients (20).

Children should stay in bed as much as possible within 6 hours after acute appendicitis resection, and the head should be sideways to prevent vomit from inhaling into respiratory tract, so as to turn over more for children, massage their waist and legs and promote blood circulation.
After 6-8 hours after operation, you can take a deep breath, turn over more, exercise your limbs, and try to get out of bed to avoid intestinal adhesion. On the second day after operation, you can eat normally. First, take liquid and semi-liquid food, and take high-protein food to promote wound healing. Eat more high-fiber fruits and vegetables after operation, and eat less gas-producing food to avoid postoperative abdominal distension (21). In recent years, there have been many reports regarding the treatment of acute appendicitis with the ERAS concept in adults at home and abroad, but there have been few reports regarding children (22-24). Therefore, the innovation of this study is to use meta-analysis method to analyze the effectiveness and safety of ERAS concept in the surgical treatment of acute appendicitis in children.

Acute appendicitis in children is a common acute abdomen, and because of the characteristics of pediatric anatomy and physiology, the disease development is very rapid, and can easily lead to the formation of appendiceal gangrene, perforation, and abscess (25). The disease is generally treated clinically by laparoscopic appendectomy. In this study, only 5 studies were included in the metaanalysis. Bagłaj et al. [2012] (26) first applied the concept of ERAS to laparoscopic acute appendectomy, but so far the concept of ERAS has not been widely used for laparoscopic acute appendectomy in children. The reason for this may be that doctors think that appendectomy can be completed with a small incision, with little trauma to children. In addition, children with small bodies require more precise models of small instruments, and the operation takes a long time and is difficult.

The results of this study showed that the postoperative incision infection rate in the ERAS group was lower than that in the control group, but there is no statistical significance $(Z=1.73$; OR $=0.56$; $95 \%$ CI: 0.29 to 1.08 ; $\mathrm{P}=0.08$ ). If it is difficult to take out the appendix, the $10 \mathrm{~mm}$ Trocar can be replaced immediately to avoid contamination of the wound successfully, and the wound need not be closed. The postoperative stay time in the ERAS group was significantly shorter than that in the control group $(\mathrm{Z}=5.26$; $\mathrm{MD}=-1.65 ; 95 \% \mathrm{CI}:-2.27$ to $-1.03 ; \mathrm{P}<0.00001)$, indicating that ERAS can significantly accelerate the recovery speed of patients and reduce the length of hospital stay, which is consistent with the findings of Pastore et al. [2014] (27). Additionally, the readmission rate and reoperation rate in RAS group were lower than those in control group $(Z=0.80$, $\mathrm{OR}=1.16,95 \%$ CI: 0.81 to $1.66, \mathrm{P}=0.42 ; \mathrm{Z}=1.14$, OR $=0.76,95 \%$ CI: 0.47 to $1.22, \mathrm{P}=0.26)$. Therefore, ERAS can 
significantly improve the quality of patient recovery and reduce the readmission rate and reoperation rate.

The results of the GRADE classification showed that $80 \%$ of the studies were of medium and high quality, indicating that the quality of the research was sufficient, the quality level of the included experiments was good, and the reliability was high. Among the 5 randomized controlled trials, 3 randomized controlled trials $(60 \%)$ described the correct method of randomization, and 1 randomized controlled trial $(20 \%)$ described the allocation concealment scheme in detail. The funnel plot showed that the circles of the included studies were basically symmetrical with the center line, suggesting that the study was of high accuracy, there was no bias in the publications, and the final conclusion was relatively credible.

\section{Conclusions}

In this meta-analysis, studies involving the application of the ERAS concept in the treatment of acute appendicitis in children as the experimental group and routine care as the control group were selected to explore the safety and efficacy of accelerated rehabilitation surgery in the perioperative treatment of acute appendicitis in children. The results of the meta-analysis confirmed that perioperative application of the ERAS concept in children with acute appendicitis can promote the rehabilitation of children, reduce the postoperative stay time, and reduce the readmission rate and reoperation rate. The shortcomings of this study lie in the small sample size and the lack of unified diagnostic criteria for each study, which might result in implementation bias. Therefore, unified diagnostic criteria should be adopted in future studies and more samples should be included to confirm the conclusions of this study. In short, with the continuous improvement of the concept of ERAS and the gradual understanding of the majority of patients and medical staff regarding ERAS, ERAS has obvious advantages over traditional routine care. However, the efficacy of ERAS in other pediatric surgeries needs to be further verified.

\section{Acknowledgments}

Funding: None.

\section{Footnote}

Reporting Checklist: The authors have completed the
PRISMA reporting checklist. Available at https://dx.doi. org/10.21037/tp-21-457

Conflicts of Interest: All authors have completed the ICMJE uniform disclosure form (available at https://dx.doi. org/10.21037/tp-21-457). The authors have no conflicts of interest to declare.

Ethical Statement: The authors are accountable for all aspects of the work in ensuring that questions related to the accuracy or integrity of any part of the work are appropriately investigated and resolved.

Open Access Statement: This is an Open Access article distributed in accordance with the Creative Commons Attribution-NonCommercial-NoDerivs 4.0 International License (CC BY-NC-ND 4.0), which permits the noncommercial replication and distribution of the article with the strict proviso that no changes or edits are made and the original work is properly cited (including links to both the formal publication through the relevant DOI and the license). See: https://creativecommons.org/licenses/by-nc-nd/4.0/.

\section{References}

1. Svensson JF, Patkova B, Almström M, et al. Nonoperative treatment with antibiotics versus surgery for acute nonperforated appendicitis in children: a pilot randomized controlled trial. Ann Surg 2015;261:67-71.

2. Benedict LA, Sujka JA, Sobrino JA, et al. Mitigating disparity in children with acute appendicitis: Impact of patient-driven protocols. J Pediatr Surg 2021;56:663-7.

3. Gardikis S, Giatromanolaki A, Kambouri K, et al. Acute appendicitis in preschoolers: a study of two different populations of children. Ital J Pediatr 2011;37:35.

4. Gauderer MW, Crane MM, Green JA, et al. Acute appendicitis in children: the importance of family history. J Pediatr Surg 2001;36:1214-7.

5. Wilmore DW, Kehlet H. Management of patients in fast track surgery. BMJ 2001;322:473-6.

6. Caruso AM, Pane A, Garau R, et al. Acute appendicitis in children: not only surgical treatment. J Pediatr Surg 2017;52:444-8.

7. Patkova B, Svenningsson A, Almström M, et al. Nonoperative treatment versus appendectomy for acute nonperforated appendicitis in children: five-year follow up of a randomized controlled pilot trial. Ann Surg 2020;271:1030-5. 
8. Willis ZI, Duggan EM, Bucher BT, et al. Effect of a Clinical Practice Guideline for Pediatric Complicated Appendicitis. JAMA Surg 2016;151:e160194.

9. Kim WJ, Jin HY, Lee H, et al. Comparing the Postoperative Outcomes of Single-Incision Laparoscopic Appendectomy and Three Port Appendectomy With Enhanced Recovery After Surgery Protocol for Acute Appendicitis: A Propensity Score Matching Analysis. Ann Coloproctol 2021;37:232-8.

10. Trejo-Ávila ME, Romero-Loera S, Cárdenas-Lailson E, et al. Enhanced recovery after surgery protocol allows ambulatory laparoscopic appendectomy in uncomplicated acute appendicitis: a prospective, randomized trial. Surg Endosc 2019;33:429-36.

11. Svensson JF, Patkova B, Almström M, et al. Outcome after introduction of laparoscopic appendectomy in children: A cohort study. J Pediatr Surg 2016;51:449-53.

12. Cundy TP, Sierakowski K, Manna A, et al. Fast-track surgery for uncomplicated appendicitis in children: a matched case-control study. ANZ J Surg 2017;87:271-6.

13. Rochon RM, Gimon T, Buie WD, et al. Expedited discharge in uncomplicated acute appendicitis: Decreasing the length of stay while maintaining quality. Am J Surg 2019;217:830-3.

14. Shafy SZ, Miller R, Uffman JC, et al. An Enhanced Recovery Protocol that Facilitates Same-day Discharge for Simple Laparoscopic Appendectomies. Pediatr Qual Saf 2019; 4:e243.

15. van Rossem CC, Schreinemacher MH, van Geloven AA, et al. Antibiotic Duration After Laparoscopic Appendectomy for Acute Complicated Appendicitis. JAMA Surg 2016;151:323-9.

16. Sherratt FC, Allin BSR, Kirkham JJ, et al. Core outcome set for uncomplicated acute appendicitis in children and young people. Br J Surg 2020;107:1013-22.

17. Yap TL, Fan JD, Ho MF, et al. Salivary biomarker for acute appendicitis in children: a pilot study. Pediatr Surg Int 2020;36:621-7.

18. Fujii T, Tanaka A, Katami H, et al. Usefulness of the pediatric appendicitis score for assessing the severity of

Cite this article as: Zhang $\mathrm{A}, \mathrm{Lu} \mathrm{H}$, Chen $\mathrm{F}, \mathrm{Wu} \mathrm{Y}$, Luo L, Sun S. Systematic review and meta-analysis of the effects of the perioperative enhanced recovery after surgery concept on the surgical treatment of acute appendicitis in children. Transl Pediatr 2021;10(11):3034-3045. doi: 10.21037/tp-21-457 acute appendicitis in children. Pediatr Int 2020;62:70-3.

19. Turel O, Mirapoglu SL, Yuksel M, et al. Perforated appendicitis in children: antimicrobial susceptibility and antimicrobial stewardship. J Glob Antimicrob Resist 2019;16:159-61.

20. Hartford EA, Woodward GA. Appendectomy or Not? An Update on the Evidence for Antibiotics Only Versus Surgery for the Treatment of Acute Appendicitis in Children. Pediatr Emerg Care 2020;36:347-52.

21. Heye P, Saavedra JSM, Victoria T, et al. Accuracy of unenhanced, non-sedated MRI in the diagnosis of acute appendicitis in children. J Pediatr Surg 2020;55:253-6.

22. Rodríguez E, Valero J, Jaramillo L, et al. Evaluation of concordance among surgeons and pathologists regarding the diagnosis and classification of acute appendicitis in children. J Pediatr Surg 2020;55:1503-6.

23. Wijayanayaka T, Davidson J, Bütter A. Does size matter? Correlation of ultrasound findings in children without clinical evidence of acute appendicitis. J Pediatr Surg 2018;53:980-3.

24. Trout AT, Sanchez R, Ladino-Torres MF. Reevaluating the sonographic criteria for acute appendicitis in children: a review of the literature and a retrospective analysis of 246 cases. Acad Radiol 2012;19:1382-94.

25. Matuszczak E, Sankiewicz A, Debek W, et al. Immunoproteasome in the blood plasma of children with acute appendicitis, and its correlation with proteasome and UCHL1 measured by SPR imaging biosensors. Clin Exp Immunol 2018;191:125-32.

26. Bagłaj M, Rysiakiewicz J, Rysiakiewicz K. Acute appendicitis in children under 3 years of age. Diagnostic and therapeutic problems. Med Wieku Rozwoj 2012;16:154-61.

27. Pastore V, Cocomazzi R, Basile A, et al. Limits and advantages of abdominal ultrasonography in children with acute appendicitis syndrome. Afr J Paediatr Surg 2014;11:293-6.

(English Language Editor: C. Betlazar-Maseh) 